\title{
Malignant Mixed Mullerian Tumor of the Uterus (Uterine Carcinosarcoma): A Case Report.
}

\author{
Dr. Siva Ranjan D. ${ }^{1}$, Dr. J Surendar ${ }^{2}$, Dr. Rama Swamy A S. ${ }^{3}$, Dr. \\ Manjunatha H K. ${ }^{4}$, \\ 1. Assistant Professor, Department of Pathology, Konaseema Institute of Medical Sciences and Research \\ Foundation, Amalapuram, India. \\ 2. Assistant Professor, Department of Forensic Medicine, Konaseema Institute of Medical Sciences and \\ Research Foundation, Amalapuram, India. \\ 3. Associate Professor, Department of Pathology, PES Institute of Medical Sciences and Research, \\ Kuppam, India. \\ 4. Associate Professor, Department of Pathology, BGS Global Institute of Medical Sciences Bangalore, \\ India.
}

\begin{abstract}
Malignant mixed mullerian tumors of the uterus (Uterine Carcinosarcomas) are rare and aggressive malignancies consisting of an epithelial (carcinoma) and a mesenchymal (sarcoma) tumor component and are considered as metaplastic endometrial carcinomas. Gebhardt in 1899 appears to have reported the first case of carcinosarcoma of uterus. Carcinosarcoma though rare, representing less than 5\% of all uterine tumors, account for $16.4 \%$ of all deaths caused by a uterine malignancy. Here, we present a case of 51 years old post menopausal women admitted to hospital with complaints of metrorrhagia and abdominal pain of 3 months duration. While hysterectomy with bilateral salpingo-oophorectomy remains the mainstay of treatment, high rates of recurrence, and metastasis suggests a need for lymphadenectomy and post operative adjuvant treatment.
\end{abstract}

KEYWORDS: Malignant Mixed Mullerian Tumor, Carcinosarcoma, Hysterectomy.

\section{INTRODUCTION}

Malignant mixed mullerian tumor (MMMT) of the uterus, also known as uterine carcinosarcomas (UC) are rare and aggressive malignancies consisting of an epithelial (carcinoma) and a mesenchymal (sarcoma) tumor component associated with poor prognosis. UC predominantly occur in postmenopausal women and a higher incidence is found among black women compared with white women ${ }^{[1]}$ synonyms for carcinosarcoma in the literature include "malignant mixed mullerian tumor," "malignant mesodermal mixed tumor" and "metaplastic carcinoma." [2]

\section{CASE HISTORY}

A 51 year old post menopausal woman admitted to hospital with complaints of metrorrhagia and abdominal pain of 3 months duration. Additionally, her complaints were spotting, vaginal bleeding, chronic constipation and headache. Physical examination revealed slight spasm on deep palpation of lower abdomen and a sense of fullness. Vaginal examination showed a normal cervix; further gynecologic examination was not carried out for fear of precipitating further bleeding. Magnetic resonance imaging (MRI) results showed a large mass in the pelvis that entirely obliterated the architecture of the uterus with endometrial cavity distension. Preoperatively limited investigations were done and patient was posted for surgery. Total abdominal hysterectomy with bilateral salpingo-oophorectomy was done and specimen was sent to histopathological examination.

\section{PATHOLOGICAL EXAMINATION}

Post-operatively we received hysterectomy specimen with bilateral fallopian tubes and ovaries separately. Specimen was fixed overnight in 10\% formalin and histopathological processing was started.

GROSS FEATURES: uterus with cervix measuring $12 \times 6 \times 4$ centimeters $(\mathrm{cm})$. Cut surface shows a large solitary polypoidal mass measuring $8 \times 4 \times 3 \mathrm{~cm}$, projecting into the uterine cavity with areas of haemorrhage and necrosis.(Fig:1) 


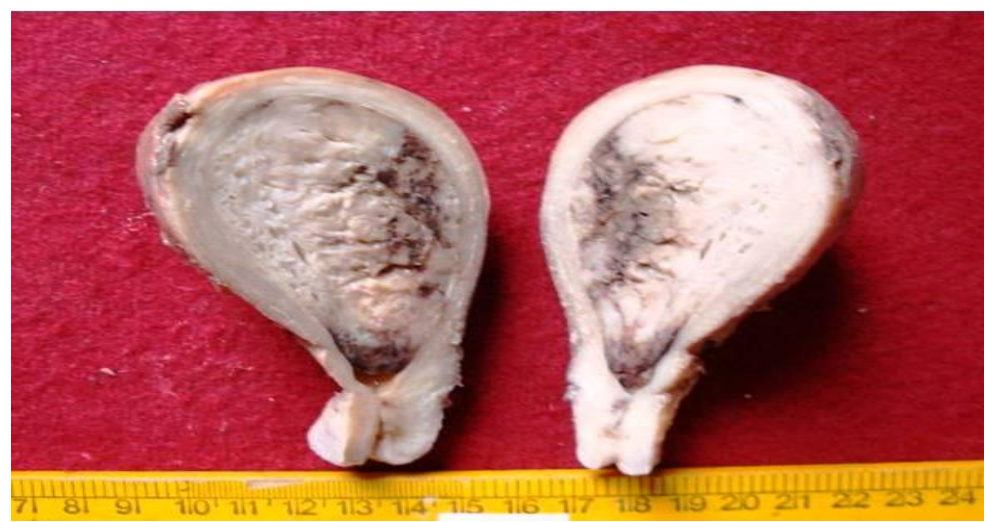

Figure 1: polypoidal mass in uterine cavity with areas of haemorrhage and necrosis.

MICROSCOPIC FINDINGS: Microscopic sections studied showed endometrial glands and stromal cells which are large, many of the nuclei are hyperchromatic having macronucleoli and few bizarre mitoses are seen. At foci showed haphazard admixture of high-grade malignant epithelial and mesenchymal components; showing pleomorphism. (Fig: 2) and at places showed homologous spindle cell sarcomatous component displaying marked pleomorphism and atypical mitotic activity. (Fig: 3 )

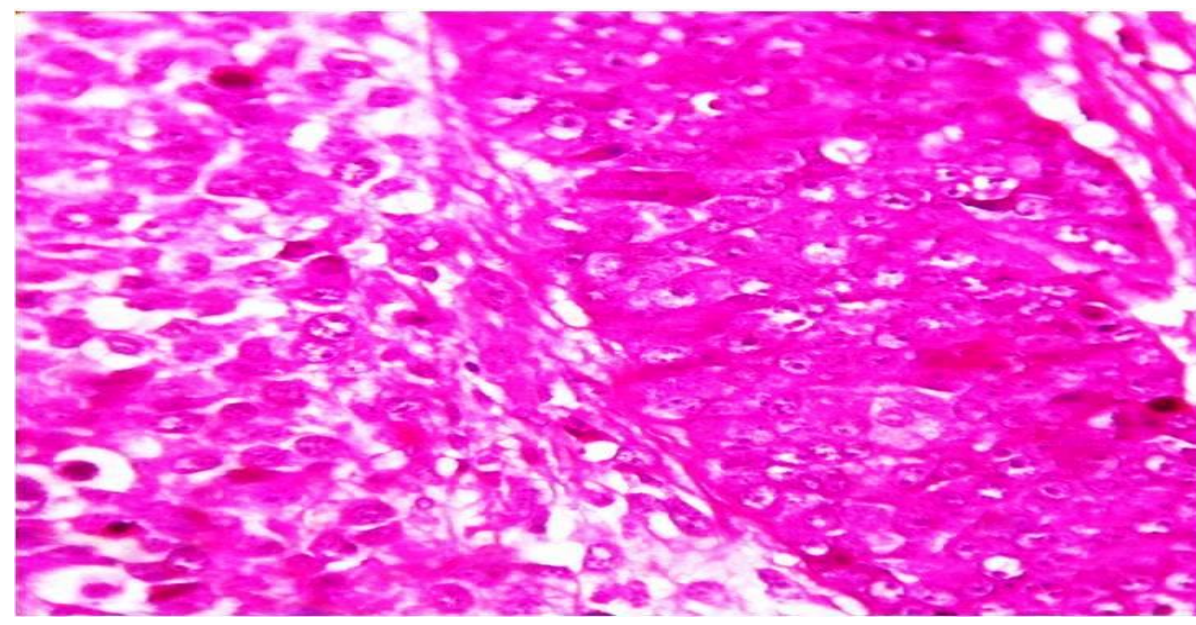

Figure 2: haphazard admixture of malignant epithelial and mesenchymal component.

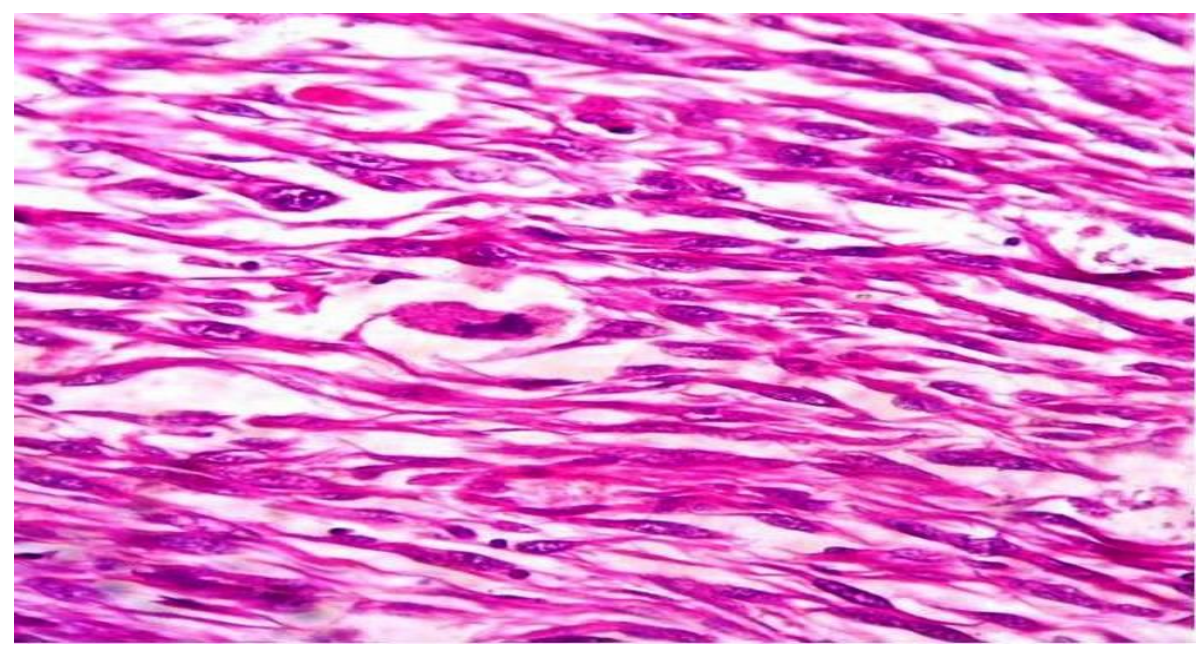

Figure 3: sarcomatous component showing bizarre atypical mitotic activity. 


\section{DISCUSSION}

Malignant uterine neoplasm's containing both carcinomatous and sarcomatous elements are designated in the World Health Organisation (WHO) classification of uterine neoplasm's as carcinosarcomas. Gebhardt in 1899 appears to have reported the first case, Meyer, after a personal examination of the slides, accepted it as authentic. ${ }^{[3]}$ Carcinosarcomas representing less than $5 \%$ of all uterine tumors, account for $16.4 \%$ of all deaths caused by a uterine malignancy. ${ }^{[4]} \mathrm{UC}$ has been identified in decreasing order of frequency in the vagina, cervix, and ovary and most rarely in the fallopian tubes. ${ }^{[5,6,7,8]}$

There are three main theories regarding the histogenesis of UC namely: ${ }^{[9,10]}$

(1) The collision theory suggests that the carcinoma and sarcoma are two independent neoplasms.

(2) The combination theory suggest that both components and derived from a single stem cell that undergoes divergent differentiation early in the evolution of the tumor.

(3) The conversion theory suggests that the sarcomatous elements derive from the carcinoma during the evolution of the tumor.

On exploring the literature we found that W G McCluggage named one more theory: The composition theory suggest that the spindle cell component is a pseudosarcomatous stromal reaction to the presence of the carcinoma. ${ }^{[11]}$

Diagnosis of UC is most often made postoperatively by histopathological examination and Immunohistochemical (IHC) studies. Microscopically UC is composed of both epithelial and mesenchymal elements may be intermittently mixed or be seen as two distinct components. ${ }^{[12]}$ The mesenchymal elements may be (a)homologous, containing cells native to the uterus including Stromal sarcoma, fibrosarcoma or leiomyosarcoma $(2 \%)$ or (b) heterologous with mixed components including rhabdomyosarcoma (18\%), chondrosarcoma (10\%), osteosarcoma $(5 \%)$ or liposarcoma $(1 \%) .{ }^{[2]}$ In our case, microscopic sections studied showed haphazardly admixed of both epithelial and mesenchymal components. The mesenchymal component showed homologous spindle cell sarcomatous component displaying marked pleomorphism and atypical mitotic activity.Several studies have found concordance of p53 staining between the carcinomatous and sarcomatous components in UC. ${ }^{[13,14]}$ IHC studies have shown that both the sarcomatous and carcinomatous components often coexpress cytokeratin and vimentin. ${ }^{[15]}$ In our case IHC studies showed p53 overexpression in both epithelial and mesenchymal component and also using antibodies against intermediate filaments, such as cytokeratin and vimentin, have shown positivity. To date; no national consensus guidelines have been established for the management of UC. ${ }^{[16]}$ While hysterectomy with bilateral salpingo-oophorectomy remains the mainstay of treatment. Recurrences occur in over half of patients after surgical treatment, ${ }^{[2]}$ high rates of recurrences and metastasis suggest a need for lymphadenectomy and post operative adjuvant treatment. In our case total abdominal hysterectomy with bilateral salpingo-oophorectomy was done; as such no recurrence was seen in our case upto one year follow up, postoperatively.

\section{CONCLUSION}

To conclude UC which arise from female genital tract can have both epithelial and mesenchymal component. If epithelial component is benign and mesenchymal component is malignant then it is called as Adenosarcoma. If both epithelial component and mesenchymal component is malignant then it is called as Carcinosarcoma. UC is a rare, highly aggressive, rapidly progressive neoplasm associated with a poor prognosis.

\section{REFERENCES}

[1] Brooks SE, Zhan M, Cote T, et al. Surveillance, epidemiology, and end results analysis of 2677 cases of uterine sarcoma 19891999. Gynecol Oncol. 2004;93:204-208.

[2] S. A. El-Nashar and A. Mariani, "Uterine carcinosarcoma," Clinical Obstetrics and Gynecology, vol. 54, 2, pp. $292-304,2011$.

[3] Meyer, R.: in Veit-Stoeckel, 6: 770-774, I930.

[4] J. S. Bosquet, S. A. Terstriep, W. A. Cliby et al., "The impact of multi-modal therapy on survival for uterine carcinosarcomas," Gynecologic Oncology, vol. 116, no. 3, pp. 419-423, 2010.

[5] A. Ahuja, R. Safaya, G. Prakash, L. Kumar, and N. K. Shukla, "Primary mixed mullerian tumor of the vagina-a case report with review of the literature," Pathology Research and Practice, vol. 207, no. 4, pp. 253-255, 2011.

[6] N. K. Sharma, J. I. Sorosky, D. Bender, M. S. Fletcher, and A. K. Sood, “ Malignant mixed mullerian tumor (MMMT) of the cervix," Gynecologic Oncology, vol. 97, no. 2, pp. 442-445, 2005.

[7] B. B. Duman, I. O. Kara, M. Gunaldi, and V. Ercolak, "Malignant mixed Mullerian tumor of the ovary with two cases and review of the literature," Archives of Gynecology and Obstetrics, vol. 283, no. 6, pp. 1363-1368, 2011.

[8] Y. M. Shen, Y. P. Xie, L. Xu et al., "Malignant mixed mullerian tumor of the fallopian tube: report of two cases and review of literature," Archives of Gynecology and Obstetrics, vol. 281, no. 6, pp. 1023-1028, 2010.

[9] R. A. de Jong, H. W. Nijman, T. F. Wijbrandi, A. K. Reyners, H. M. Boezen, and H. Hollema, "Molecular markers and clinical behavior of uterine carcinosarcomas: focus on the epithelial tumor component," Modern Pathology. In press.

[10] Z. Jin, S. Ogata, G. Tamura et al., "Carcinosarcomas (malignant mullerian mixed tumors) of the uterus and ovary: a genetic study with special reference to histogenesis," International Journal of Gynecological Pathology, vol. 22, no. 4, pp. 368-373, 2003. 
[11] W.G. McCluggage, Malignant biphasic uterine tumors: carcinosarcomas or metaplastic carcinomas?. J Clin Pathol. 2002; 55:321-5.

[12] L. Brown, "Pathology of uterine malignancies," Clinical Oncology, vol. 20, no. 6, pp. 433-447, 2008.

[13] Mayall F, Rutty K, Campbell F, et al. p53 immunostaining suggests that uterine carcinosarcomas are monoclonal. Histopathology 1994:24:211-14.

[14] Szukala SA, Marks JR, Burchette JL, et al. Coexpression of p53 by epithelial and stromal elements in carcinosarcoma of the female genital tract: an immunohistochemical study of 19 cases. Int J Gynecol Cancer 1999;9:131-6.

[15] George E, Manivel JC, Dehner LP, et al. Malignant mixed Mullerian tumors: an immunohistochemical study of 47 cases, with histogenetic considerations and clinical correlation. Hum Pathol 1991;22:215-23.

[16] A. V. Genever and S. Abdi, "Can MRI predict the diagnosis of endometrial carcinosarcoma?"Clinical Radiology, vol. 66, no. 7, pp. 621-624, 2011. 